\title{
XVI. On the hygrometer by evaporation
}

\author{
J. Ivory M.A. F.R.S.
}

To cite this article: J. Ivory M.A. F.R.S. (1822) XVI. On the hygrometer by evaporation, Philosophical Magazine Series 1, 60:292, 81-88, DOI: 10.1080/14786442208652797

To link to this article: http://dx.doi.org/10.1080/14786442208652797

曲 Published online: 29 Jul 2009.

Submit your article to this journal $\pi$

LII Article views: 4

Q View related articles $₫$

4 Citing articles: 2 View citing articles 


\title{
PHILOSOPHICAL MAGAZINE AND JOURNAL.
}

\author{
$31^{\text {st }} A U G U S T 1822$.
}

XVI. On the Hygrometer by Eraporation. By J. Ivory,
M.A. F.R.S.

To the Editors of the Philosophical Magazine and Journal. Gentremen,

1. $\mathbf{T}_{\mathrm{HE}}$ idea of employing the evaporating power of the atmosphere for discovering its condition in regard to moisture, is due to the late most ingenious philosopher Dr. James Hutton of Edinburgh. He dipped a thermometer in water, both being previously brought to the general temperature; and then exposing the wet bulb to a current of air, he marked how many degrees the mercury sunk in the tube; and he estimated the dryness of the air by the quantity of the depression. This is certainly a very curious experiment; and if we go back to the time at which it was made, before 1792, it will perhaps be admitted that no accurate and complete account of it could then be given, for want of a sufficient knowledge of the laws that obtain between permanent elastic fluids and the vapours mixed with them. The experiment is deserving of discussion chiefly in two respects:-Is the degree of the depression of the thermometer dependent only on the quantity of moisture in the atmosphere, without being affected by accidental circumstances? What is the relation between the depression of the thermometer and the tension of the aqueous vapour diffused in the atmosphere?

Professor Leslie, of Edinburgh, has determined that the cold produced in Dr. Hutton's experiment is owing entirely to the drying quality of the atmosphere, and that it is not influenced by any other circumstances; and applying that very delicate instrument, his differential thermometer, to measure the degree of depression, he has constructed the hygrometer that bears his name. This instrument has been many years before the public, and it has been too ably described by its learned inventor, in many different publications, to require any further development of its principles or properties. On the present occasion our intention reaches no further than to discuss the original experiment made by Dr. Hutton.

Vol.60. No. 292. Aug. 1822.

L

2. We 
2. We may suppose two different thermometers; one for marking the general temperature; and the other, having its bulb dipped in water, or covered with a wet linen rag, or with bibulous paper soaked in water, for the purpose of ascertaining the cold produced by the evaporating power of the air. Now, if we suppose that the atmosphere is saturated with moisture, its contact with the wet bulb will produce neither any evaporation from the humid surface, nor any deposition of moisture upon it from the air. As there is no action between the air and the bulb, neither heat nor cold will be produced; and the mercury will stand at the same height in both thermometers. But if the air be not saturated with moisture, there will be an evaporation from the wet bulb; and, evaporation being invariably attended with the disappearance of heat of temperature which enters into the constitution of the vapour in a latent form, the mercury of the thermometer will be depressed. The air in contact with the moist surface will therefore become damper both by the depression of its temperature and by the small addition of humidity diffused in it. If , in a single instant of time, the point of saturation with humidity be not fully attained, the same process will be repeated in a second and a third instant, until at length the air is charged with all the vapour it is capable of retaining at its depressed temperature.

It is certain that the thermometer cannot be depressed below the point at which the air is saturated with humidity. Even if, by any accidental cause, we suppose a greater degree of cold to be produced, the consequence would be a deposition of part of the vapour in the liquid form, attended with an evolution of heat; by which means, supposing the extraneous cooling cause to be removed, the thermometer would again rise and settle at the point of saturation.

In perfectly still air it is known that evaporation goes on very slowly; and this circumstance may make it difficult to discern when the action between the air and the bulb has come to an invariable state. Some agitation of the instrument, or some motion in the air, seems to be proper, whether to hasten the permanent degree of cold, or to ascertain that it has actually taken place.

On the other hand, every portion of air must take up some definite time in parting with its heat and absorbing its share of vapour; and if we suppose a circulation so brisk as to displace the air from the thermometer in half that time, it is plain that it would be only half saturated with moisture by its contact with the wet bulb; in which case the experiment would be unsuccessful. This however is only an extreme case, which 
it would be easy to guard against, were the effect well ascertained by experiment.

Another disturbing cause it seems difficult entirely to obviate. For the stem of the thermometer, having constantly the general temperature of the atmosphere, will communicate some of its heat to the colder bulb; and although the air which touches the bulb may in this case be fully saturated with humidity, yet the heat extricated from it in cooling will be less than the latent heat of the vapour diffused in it: whereas the success of the experiment requires an exact equality between these two quantities of heat.

In this experiment it is presumed that all the air that comes in contact with the wet bulb is continually saturated with vapour, and likewise that all the heat necessary for feeding the evaporation is furnished by the same air. If the air be displaced from the humid surface without being saturated with moisture; or if the heat that enters in a latent form into the constitution of the rising vapour, be derived from any other source than the air that comes successively into contact with the wet bulb; in both these cases the experiment will fail as a means of discovering the state of the atmosphere in regard to moisture. The effects of the disturbing causes that have been enumerated, do not appear to be so formidable as to endanger the success of the experiment to a degree of accuracy sufficient for most practical purposes.

3 . If we now suppose that the temperature of the thermometer is stationary, and that every portion of air becomes saturated with moisture by its contact with the wet bulb, and likewise that it deposits just as much of its heat as is contained in a latent form in the vapour it absorbs; we are led to this question:

Pros. A given quantity of air, as a cubic foot, under a given pressure, having a given temperature, and containing vapour of some unknown tension, being supposed to have its temperature depressed by any cause, and likewise to have the vapour due to all the heat extricated in cooling continually diffused in it, till at length it becomes saturated with moisture at some known temperature; it is required to find the unknown tension of the aqueous vapour contained in the air.

By the vapour due to a certain quantity of heat is meant, the vapour which contains the proposed heat in a latent form; and it is supposed that the same quantity of heat is absorbed in the formation of vapour at all temperatures.

To solve this problem, let $b$ denute the height of the barometer, $\tau$ the temperaturc estimated on the centigrade scale, I. 2 and 
and $x$ the unknown tension of the aqueous vapour: then the weight of a cubic foot of the atmospheric air will, in these circumstances, be equal to the weight of a cubic foot of dry air under the pressure $b-x$, and the weight of a cubic of vapour of the tension $x$, the common temperature being $\tau$.

Let $w$ stand for the weight of a cubic foot of vapour at the temperature zero, the tension, as determined by Dalton, being $\frac{1}{5}$ of an inch of the barometric column: then the weight of a cubic foot of dry air at the same temperature and pressure will be $\frac{8}{5} \times w^{*}$; and consequently the weight of a cubic foot of the same air at the same temperature and under the pressure 30 inches, will be $5 \times 30 \times \frac{3}{5} \times w$, or $240 \times w$.

Now, $240 \times$ w being the weight of a cubic foot of dry air at the temperature zero and pressure 30 inches, the weight of the same volume when the temperature is $\tau$, and the pressure $b-x$, will be

$$
240 \times w \times \frac{b-x}{30} \times \frac{1}{1+m r}
$$

where $m=\cdot 00375$, or the expansion for one centesimal degree: and the weight of a cubic foot of vapour of the temperature $\tau$, and tension $x$, will be $5 w \times \frac{x}{1+n r}$. Wherefore the weight of a cubic foot of atmospheric air originally considered will be thus expressed, viz.

$$
240 w \times \frac{b-x}{30} \times \frac{1}{1+m r}+5 w \times \frac{x}{1+m r} .
$$

Let $a$ stand for the specific heat of air, and $s$ for that of vapour: then we get

$$
\left\{240 w \times \frac{a}{1+m \tau} \times \frac{b-x}{30}+5 w \times \frac{x \cdot s}{1+m \tau}\right\} \times \varepsilon \tau
$$

for the weight of water that would be raised one degree of the thermometer, by the heat extricated in cooling the mixed mass of air and vapour a number of degrees expressed by $\delta \tau$.

$A$ gain, put $\lambda$ for the latent heat of steam: then

$$
\left\{240 \times \frac{a}{1+m_{r}} \times \frac{b-x}{30}+5 w \times \frac{x^{*} s}{1+m_{r}}\right\} \times \frac{d r}{\lambda}
$$

will be the weight of steam due to the above-mentioned quantity of heat; or the weight of vapour that contains in a latent state all the heat extricated in cooling the mass of air to the temperature $\tau-\delta \tau$.

Wherefore we have the following weight of dry air and var

* Biot's Traité de Physique, vol. i. pp. 996, 297 . 
pour at the temperature $\tau-\delta \tau$, instead of the original cubic foot of mixed air, viz.

$$
\begin{aligned}
240 \times \frac{1}{1+m r} \times \frac{b-x}{30}+5 w \times \frac{x}{1+m r} & \\
+ & \left\{240 w \times \frac{a}{1+m r} \times \frac{b-x}{30}+5 w \times \frac{x \cdot s}{1+m r}\right\} \times \frac{\delta r}{\lambda},
\end{aligned}
$$

the first term only being air, and the rest vapour.

The air at the reduced temperature $\tau-\delta \tau$, and with its new accession of humidity, is in a state of saturation. Its tension $\rho$ will therefore depend only on the temperature $\tau-\delta \tau$; insomuch that when one of these quantities is given, the other may be thence found by means of the Tables and formulæ usually given in this part of natural philosophy. Now at the temperature $\tau-\varepsilon \tau$, and under the pressure $b$, the weight of a cubic foot of air mixed with vapour at the tension $\varrho$, will be equal to,

$$
240 w \times \frac{1}{1+m(\tau-\dot{\partial} r)} \times \frac{b-e}{30}+5 w \times \frac{e}{1+m(\tau-\delta, r)},
$$

the first term being air, and the second vapour.

But, in any two parcels of the same air, there must be the same proportion between the two constituent parts. I therefore divide the air in the expression (A) by the air in the expression (B); and likewise the vapour of the former expression by the vapour of the latter: and as the two quotients must be equal, I get this equation, viz.

$$
\frac{b-x}{b-e}=\frac{5 x+\left\{240 a \times \frac{b-x}{30}+5 x\right\}}{5 e} \cdot \frac{\delta \tau}{\lambda}=
$$

which, by the usual reduction, becomes

$$
x \cdot\left\{1+\left(s-\frac{8 a}{5}\right) \cdot\left(1-\frac{e}{b}\right) \cdot \frac{\delta r}{\lambda}\right\}=\left\{\left\{1+\frac{8 a}{5} \cdot \frac{\delta r}{\lambda}\right\}-\frac{b}{50} \cdot \frac{48 a \cdot \partial r}{\lambda} .\right.
$$

Now in the 4th vol. p. 726, of Biot's Traite' de Physique, I find $a=\cdot 2669=\frac{t}{15}$ nearly, $s=\cdot 847=\frac{17}{2}$ nearly, and, at p. 713, $\lambda=550:$ and, by substituting these numbers, it will be found that the coefficient of $x$ is very nearly equal to that of $\varrho$ : the same coefficients also are both little different from unit, because $\delta \tau$ is always only a small part of $\lambda$ : and, by means of these considerations, the foregoing equation may be simplified without detracting almost in any degree from its exactness, viz.

$$
x=\rho-\frac{b}{30} \times \frac{48 a, 5 r}{\lambda} \text {. }
$$

Further, because $\frac{b}{30}$ is in most cases very nearly equal to unit, ne get

$$
x=f-\frac{4 c \alpha \cdot d r}{\lambda}
$$

that 
that is, in words, "The actual tension of the vapour in the atmosphere is less than the maximum tension at the reduced temperature $\tau-\delta \tau$, by a quantity proportional to the observed depression."

If we were in possession of any different method of finding the tension of the vapour in the atmosphere, we should be able both to correct the coefficient of $\delta \tau$ in the foregoing formula, which number is liable to some uncertainty, and likewise to judge of the degree of accuracy of which Dr. Hutton's experiment is susceptible. Now such a method of experimenting has been employed by Dalton. It consists in exposing to the air a clean and dry surface of glass cooled artificially till it is observed that moisture begins to be deposited on it. When this takes place, the air in contact with the cold surface is in a state of saturation; and, the temperature of the dewing point being thus determined, it is easy to deduce from it the tension of the vapour at the actual temperature of the atmosphere.

Let $y$ denote the weight of vapour contained in a cubic foot of the air at the general temperature $\tau$; and $h$, the weight of vapour in a cubic foot at the reduced temperature $\tau-\delta \tau$; then, according to what has already been shown, we have

And hence,

$$
\begin{aligned}
& y=\frac{5 w}{1+m \tau} \times x, \\
& h=\frac{5 w}{1+m(\tau-\delta \tau)} \times g ;
\end{aligned}
$$

$$
\begin{aligned}
& x=\frac{y}{5 w} \times(1+m \tau) \\
& \varrho=\frac{h}{5 w} \times(1+m \tau) \times(1-m \delta \tau) ;
\end{aligned}
$$

and, by substituting these values in the formula (2), we get

and, by omitting insensible quantities,

$$
y=h(1-m \delta \tau)-\frac{240 a w}{\lambda} \times \frac{\delta \sigma}{1+m \tau}
$$

$$
y=h-\frac{240 a w}{\lambda} \times \delta \tau
$$

or, in words, "The quantity of vapour in a cubic foot of air at the actual temperature $\tau$, is less than the maximum of humidity which the air is capable of retaining at the reduced temperature $\tau-\delta \tau$, by a quantity proportional to the observed depression."

. If we denote by $\mathbf{H}$ the maximum of humidity in a cubic foot of the air at the temperature $\tau$; then

$$
\mathrm{H}-h+\frac{240 a w}{\lambda} \times 8 \tau \text {, }
$$


will express how much the vapour really contained in the air falls short of saturation; and this seems to be the proper hygrometric quantity, or the just measure of the drying quality of the atmosphere.

It is proper to observe that the formula for computing $\mathrm{H}$ when $\tau$ is given, is

$$
\text { Log. } \mathrm{H}=\mathrm{A} \tau+\mathrm{B} \tau^{2}+\& \mathrm{c} \text {; }
$$

whence it follows that $\mathrm{H}-h$ is not proportional to $\delta \tau$, but to $H \times \delta \tau$. The measure of the dryness of the air is therefore a quantity which varies both with the observed depression and the actual humidity of the atmosphere.

It appears therefore that the experiment of Dr. Hutton will fully disclose the condition of the atmosphere with regard to moisture. Nothing more is necessary for this purpose than to provide a table containing, in one column, the maximum tensions of the vapour for all degrees of temperature, such as is found in vol. 1. of Biot's Traité de Physique; and, in another column, the weights of vapour in a given volume, that correspond to the different tensions; quantities which, for every tension $\varrho$ and temperature $\tau$, are computed by the formula, $5 w \times \frac{\rho}{1+m r}$.

4. There is a great analogy between the experiment of Dr. Hutton and the method employed by Dalton for finding the Dewing point. In the latter method the air is brought to a state of saturation by the agency of cold alone; in the former the same effect is produced by the joint operation of cold and the addition of humidity. But Dalton's process cannot be used without some complication of machinery and the employing of artificial means for producing cold; whereas Dr. Hutton's experiment requires no more than the exposing of the wet bulb of a thermometer to the action of the atmosphere. In the one case, great nicety of observation is required for detecting the precise moment when the dew begins to be deposited; and from this cause considerable uncertainty, it is natural to think, must often arise, more especially at low temperatures, when the air contains only a very small portion of humidity. In the other case, the observer has only to read off the degree on the scale of a thermometer; and it appears that any want of precision in the experiment will cause only a proportional inaccuracy in the result; insomuch that the quantity of moisture in the atmosphere may be known with an exactness sufficient for most practical purposes, even if we suppose an uncertainty of half a degree, or a whole degree, in the quantity of the depression. The simplicity of the one process fits it for being useful in many situations, (in an astronomical 
observatory for instance) where the complication attending the other would render it quite inapplicable.

5. If we use the numbers formerly noticed for $a$ and $\lambda$, and put $w=2.3$ troy grains; then $\frac{48 a}{\lambda}=0233$, or $\frac{1}{10}$ nearly; and $\frac{48 a w}{\lambda}=26$, or $\frac{1}{4}$ nearly; and the formulæ (2) and (3) will become

$$
\begin{aligned}
& x=\rho-\frac{8 x}{40}, \\
& y=m-\frac{8 x}{4} .
\end{aligned}
$$

These expressions are very simple and easily remembered. If to the scale of the evaporating thermometer another scale were attached, showing the maximum tensions at the various degrees of temperature; or one showing the maximum quantities of humidity in a cubic foot at the same temperatures; we should only have to deduct $\frac{1}{40}$ of the observed depression to have the actual tension sought, or $\frac{1}{4}$ of the same quantity, to have the actual quantity of vapour in a cubic foot. As $\delta \tau$ will always be confined to a few degrees, a small table containing the quantities to be subtracted might be engraved apart on the scale of the instrument.

The purpose of these observations is to explain the method proposed by Dr. Hutton for finding the moisture of the atmosphere on its own principles, and to disengage it from all mechanism foreign to it. The process requires nothing more than two thermometers which we have supposed to be constructed according to the centigrade scale. It is greatly to be wished that a number of experiments were made sufficient for ascertaining the practical fitness of the method for accomplishing its end.

J. IVoRY.

XVII. On a Nere Theory of the Tides. By Capt. Forman, of the Royal Navy.

To the Editors of the Philosophical Magazine and Journal.

Gentlemen, - $\mathbf{I}_{\mathrm{F}}$ the facts and arguments contained in the following letter are true, they must add to our stock of knowledge, and ought to be made known; if they are not, they can easily be refuted; and therefore, as in either case they can do no harm, I shall feel obliged if you will find room for them in your Magazine. I am, gentlemen, \&c.

W. Fonmana 